SCIENCE CHINA

Physics, Mechanics \& Astronomy

\title{
A new trend in nuclear physics development
}

\author{
Bing Guo, and NaiYan Wang* \\ Department of Nuclear Physics, China Institute of Atomic Energy, Beijing 102413, China
}

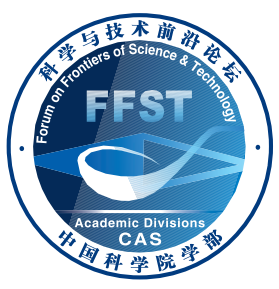

Received June 7, 2017; accepted June 19, 2017; published online July 27, 2017

\begin{abstract}
Citation: $\quad$ B. Guo, and N. Y. Wang, A new trend in nuclear physics development, Sci. China-Phys. Mech. Astron. 60, 102031 (2017), doi: 10.1007/s11433-0179075-1
\end{abstract}

Atomic nuclei comprise a principal level of material structure. Nuclear physics, in itself an substantial area of study, serves as a link between atomic physics and particle physics. The main research areas of nuclear physics involve strong interaction, weak interaction, and electromagnetic interaction.

Nuclear physics, a vital frontier in the development of physics in the present century, is now moving in two important developmental directions [1,2]. One direction includes the theory of strong interaction and the standard model, both of which are cutting-edge subjects and are of common interest in nuclear physics and particle physics. The essential role of nuclear physics in this direction includes providing information on relativistic heavy ion collisions $[3,4]$, interactions between high-energy continuous electron beams and atomic nuclei [5], and interactions between high-energy hadrons and nuclei. The other direction includes explorations of the behaviors of atomic nuclei far away from the stable line (Figure 1 [6]) [7], examining nuclear theory based on the data along the stable line, observing the recently discovered doubly-magic nuclei [8], finding a new path for synthesis of heavy elements in the universe (Figure 2 [9]) [10,11], and studying single-particle energy levels, residual interactions, and multi-body correlations. In addition, new super-deformations and new radioactive decay will be explored and standard models will be re-examined.

Many of the experimental techniques and theoretical methods developed in the study of nuclear physics have been widely applied to other areas within modern physics. Nuclear physics and other disciplines combine to form many interdisciplinary areas of study, for example nuclear astrophysics.

The field of nuclear energy, including fission and fusion energies, is central to the applications of nuclear research that seek to develop sustainable and clean energy resources. Fission energy has become a major energy source which is necessary to economic and social developments. In order to solve energy problems more effectively, almost 50 years of study
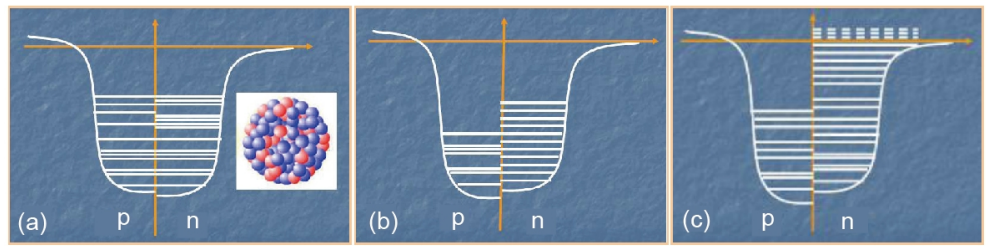

Figure 1 Diagram of nuclei from stability line to drip line. (a) Stable nuclei, (b) unstable nuclei, (c) drip line nuclei [6].

*Corresponding author (email: wangny@bnu.edu.cn) 


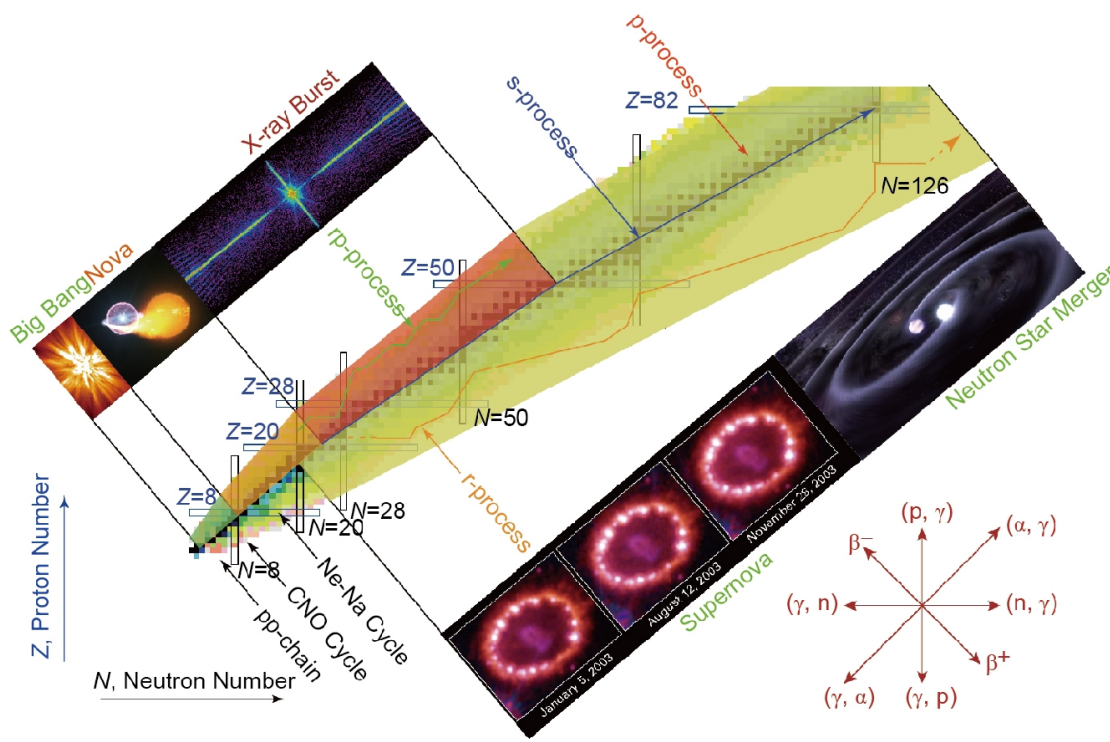

Figure 2 Map of atomic nuclei and nucleosynthesis processes occurred in the universe [9].

have been devoted to obtaining more abundant and ideal fusion energy. In addition, nuclear technology has been widely used in medicine, industry, agriculture, and environmental protection, as well as in other fields. Development of nuclear physics, nuclear technology, nuclear industry, and related support industries, have created a system that underpins both China's energy industry and national defense.

This work was supported by the Consultation Project of the Academic Divisions of the Chinese Academy of Sciences (CASAD), and the National Natural Science Foundation of China (Grant No. L1524018).

1 R. Tribble, D. Bryman, D. Dean, C. Elster, R. Ent, T. Glasmacher, U. Heinz, X. Ji, R. Lacey, I. Lee, N. Makins, R. Milner, M. Ramsey-Musolf, H. Nitsche, G. Savard, S. Seestrom, T. Ulrich, U. Kolck, J. Wilkerson, and W. Zajc, Report of The Frontiers of Nuclear Science, Long range plan (Department of Energy and National Science Foundation, 2007).

2 G. Rosner, F. Azaiez, M. Borge, A. Bracco, G. Cata-Danil, C. Philippe, R. Čaplar, J. Dobeš, A. Eiró, J. Gaardhøje, S. Harissopulos, P. Heenen, T. Johansson, A. Jokinen, K. Jungmann, B. Krusche, E. Nappi, P. Nolan, T. Peitzmann, A. Richter, D. Röhrich, C. Roy, H. Stöcker, H. Ströher, J. Styczeń, J. Wambach, E. Widmann, and G. Wolf, Report of Perspectives of Nuclear Physics in Europe, NuPECC Long range plan (European Science Foundation, 2010).

3 J. Adams, et al. (STAR Collaboration), Nucl. Phys. A 757, 102 (2005).

4 K. Adcox, et al. (PHENIX Collaboration), Nucl. Phys. A 757, 184 (2005).

5 D. Boer, M. Diehl, R. Milner, R. Venugopalan, W. Vogelsang, A. Accardi, E. Aschenauer, M. Burkardt, R. Ent, V. Guzey, D. Hasch, K. Kumar, M. A. C. Lamont, Y. Li, W. J. Marciano, C. Marquet, F. Sabatie, M. Stratmann, F. Yuan, S. Abeyratne, S. Ahmed, C. Aidala, S. Alekhin, M. Anselmino, H. Avakian, A. Bacchetta, J. Bartels, H. BC, J. Beebe-Wang, S. Belomestnykh, I. Ben-Zvi, G. Beuf, J. Blumlein, M . Blaskiewicz, A. Bogacz, S. J. Brodsky, T. Burton, R. Calaga, X. Chang, I. O. Cherednikov, P. Chevtsov, G. A. Chirilli, C. Ciofi degli
Atti, I. C. Cloet, A. Cooper-Sarkar, R. Debbe, Ya. Derbenev, A. Deshpande, F. Dominguez, A. Dumitru, R. Dupre, B. Erdelyi, C. Faroughy, S. Fazio, A. Fedotov, J. R. Forshaw, R. Geraud, K. Gallmeister, L. Gamberg, J.-H. Gao, D. Gassner, F. Gelis, G. P. Gilfoyle, G. Goldstein, K. Golec-Biernat, V. P. Goncalves, M. Gonderinger, M. Guzzi, P. Hagler, H. Hahn, L. Hammons, Y. Hao, P. He, T. Horn, W. A. Horowitz, M. Huang, A. Hutton, B. Jager, W. Jackson, A. Jain, E. C. Johnson, Z. B. Kang, L. P. Kaptari, D. Kayran, J. Kewisch, Y. Koike, A. Kondratenko, B. Z. Kopeliovich, Y. V. Kovchegov, G. Krafft, P. Kroll, S. Kumano, K. Kumericki, T. Lappi, T. Lautenschlager, R. Li, Z. T. Liang, V. N. Litvinenko, S. Liuti, Y. Luo, D. Muller, G. Mahler, A. Majumder, S. Manikonda, F. Marhauser, G. McIntyre, M. Meskauskas, W. Meng, A. Metz, C. B. Mezzetti, G. A. Miller, M. Minty, S. O. Moch, V. Morozov, U. Mosel, L. Motyka, H. Moutarde, P. J. Mulders, B. Musch, P. Nadel-Turonski, P. Nadolsky, F. Olness, P. N. Ostrumov, B. Parker, B. Pasquini, K. Passek-Kumericki, A. Pikin, F. Pilat, B. Pire, H. Pirner, C. Pisano, E. Pozdeyev, A. Prokudin, V. Ptitsyn, X. Qian, J. W. Qiu, M. Radici, A. Radyushkin, T. Rao, R. Rimmer, F. Ringer, S. Riordan, T. Rogers, J. Rojo, T. Roser, R. Sandapen, R. Sassot, T. Satogata, H. Sayed, A. Schafer, G. Schnell, P. Schweitzer, B. Sheehy, J. Skaritka, G. Soyez, M. Spata, H. Spiesberger, A. M. Stasto, N. G. Stefanis, M. Strikman, M. Sullivan, L. Szymanowski, K. Tanaka, S. Taneja, S. Tepikian, B. Terzic, Y. Than, T. Toll, D. Trbojevic, E. Tsentalovich, N. Tsoupas, K. Tuchin, J. Tuozzolo, T. Ullrich, A. Vossen, S. Wallon, G. Wang, H. Wang, X. N. Wang, S. Webb, C. Weiss, Q. Wu, B. W. Xiao, W. Xu, B. Yunn, A. Zelenski, Y. Zhang, J. Zhou, and P. Zurita, arXiv: 1108.1713v2.

6 B. Guo, and W. P. Liu, Sci. Bull. 60, 1820 (2015).

7 O. Sorlin, and M. G. Porquet, Prog. Particle Nucl. Phys. 61, 602 (2008), arXiv: 0805.2561.

8 D. Steppenbeck, S. Takeuchi, N. Aoi, P. Doornenbal, M. Matsushita, H. Wang, H. Baba, N. Fukuda, S. Go, M. Honma, J. Lee, K. Matsui, S. Michimasa, T. Motobayashi, D. Nishimura, T. Otsuka, H. Sakurai, Y. Shiga, P. A. Söderström, T. Sumikama, H. Suzuki, R. Taniuchi, Y. Utsuno, J. J. Valiente-Dobón, and K. Yoneda, Nature 502, 207 (2013).

9 B Guo, W Liu, Z Li, Nuclear Astrophysics (China Atomic Energy Press, Beijing, 2017), p. 28.

10 M. Arnould, S. Goriely, and K. Takahashi, Phys. Rep. 450, 97 (2007), arXiv: 0705.4512 .

11 M. Arnould, and S. Goriely, Phys. Rep. 384, 1 (2003). 